\title{
Transversal Structures on Triangulations, with Application to Straight-Line Drawing
}

\author{
Éric Fusy \\ Algorithm Project (INRIA Rocquencourt) and LIX (École Polytechnique) \\ eric.fusy@inria.fr
}

\begin{abstract}
We define and investigate a structure called transversal edgepartition related to triangulations without non empty triangles, which is equivalent to the regular edge labeling discovered by Kant and He. We study other properties of this structure and show that it gives rise to a new straight-line drawing algorithm for triangulations without non empty triangles, and more generally for 4-connected plane graphs with at least 4 border vertices. Taking uniformly at random such a triangulation with 4 border vertices and $\mathrm{n}$ vertices, the size of the grid is almost surely $\frac{11}{27} n \times \frac{11}{27} n$ up to fluctuations of order $\sqrt{n}$, and the halfperimeter is bounded by $n-1$. The best previously known algorithms for straight-line drawing of such triangulations only guaranteed a grid of size $(\lceil n / 2\rceil-1) \times\lfloor n / 2\rfloor$. Hence, in comparison, the grid-size of our algorithm is reduced by a factor $\frac{5}{27}$, which can be explained thanks to a new bijection between ternary trees and triangulations of the 4-gon without non empty triangles.
\end{abstract}

\section{Introduction}

A plane graph is a connected graph embedded in the plane so that edges do not cross each other. Many algorithms for drawing plane graphs [4, 15, 2, 10, endow the graph with a particular structure, from which it is possible to give coordinates to vertices in a natural way. For example, triangulations, i.e., plane graphs with only faces of degree 3, are characterized by the fact that their inner edges can essentially be partitioned into three spanning trees, called Schnyder Woods, with specific incidence relations [15]. Using these spanning trees it is possible to associate coordinates to each vertex by counting faces on each side of particular paths passing by the vertex. Placing vertices in this way and linking adjacent vertices by segments yields a straight-line drawing algorithm, which can be refined to produce a drawing on a regular grid of size $(n-2) \times(n-2)$, see 16 .

A plane graph with an outer face of degree $k$ and inner faces of degree 3 is called a triangulation of the $k$-gon. If the interior of any 3 -cycle of edges is a face, the triangulation is irreducible. Observe that it implies $k>3$, unless the graph is reduced to a unique triangle. There exist more compact straight-line drawing algorithms for irreducible triangulations [9, 11], the size of the grid being guaranteed to be $(\lceil n / 2\rceil-1) \times\lfloor n / 2\rfloor$ in the worst case. 
In this extended abstract we concentrate on irreducible triangulations of the 4-gon, which carry a good level of generallity. Indeed many graphs, including 4-connected plane graphs with at least 4 border vertices, can be triangulated (after adding 4 vertices in the outer face) into an irreducible triangulation of the 4-gon, see 1. By investigating a bijection with ternary trees, we have observed that each irreducible triangulation of the 4-gon can be endowed with a structure, called transversal edge-partition, which can be summarized as follows. Calling $S_{b}, N_{r}, N_{b}, S_{r}$ (like south-blue, north-red, north-blue, southred) the 4 border vertices of $T$ in clockwise order, the inner edges of $T$ can be oriented and partitioned into two sets: red edges that "flow" from $S_{r}$ to $N_{r}$, and blue edges that "flow" from $S_{b}$ to $N_{b}$. For those familiar with bipolar orientations [5], i.e. acyclic orientations with two poles, the structure can also be seen as a transversal couple of bipolar orientations, see Section 2.3. As we learned after completing a first draft of this extended abstract, Kant and He used an equivalent structure in [10] and derived nice algorithms of rectangular-dual drawing and of visibility representation. We explore the properties of this structure and show in particular in Theorem 1 that it is of the lattice type.

In Section 3, we derive from the transversal structure a straight-line drawing algorithm of an irreducible triangulation $T$ of the 4-gon. Like drawing algorithms using Schnyder Woods [15,2, it is based on face counting operations. The first step is to endow $T$ with a particular transversal edge-partition, said minimal, which is obtained by application of an iterative algorithm described in Section 2.4. Then the transversal structure is used to associate to each vertex $v$ a path $P_{r}$ of red edges and a path $P_{b}$ of blue edges, both passing by $v$. The abscissa (resp. ordinate) of $v$ is obtained by counting faces on each side of $P_{r}$ (resp. $P_{b}$ ). Our algorithm outputs a straight line embedding on a regular grid of width $W$ and height $H$ with $W+H \leq n-1$ if the triangulation has $n$ vertices. This algorithm can be compared to [9] and [11, which produces straight-line drawing on a grid of size $(\lceil n / 2\rceil-1) \times\lfloor n / 2\rfloor$. However, algorithms of [9] and [1] rely on a particular order of treatment of vertices called canonical ordering, and a step of coordinate-shifting makes them difficult to implement and to carry out by hand. As opposed to that, our algorithm can readily be performed on a piece of paper, because coordinates of vertices can be computed independently with simple face-counting operations. Finally, our algorithm has the nice feature that it respects the structure of transversal edge-partition. Indeed, Theorem 2 ensures that red edges are geometrically oriented from $S_{r}$ to $N_{r}$ and blue edges are geometrically oriented from $S_{b}$ to $N_{b}$.

A compact version of the algorithm even ensures that, for a random triangulation with $n$ vertices, the size of the grid is asymptotically almost surely $\frac{11}{27} n \times \frac{11}{27} n$ up to small fluctuations, of order $\sqrt{n}$. Compared to [9] and [11], we do not improve on the size of the grid in the worst case, but improve asymptotically by a reduction-factor $5 / 27$ on the width and height of the grid for a typical (random) object of large size, see Figure 4.2 for an example with $n=200$. The reduction factor $5 / 27$ can be explained thanks to a new bijection between ternary 
trees and irreducible triangulations of the 4-gon. This bijection is described in Section 4 and relies on "closure operations", as introduced by G. Schaeffer [13, see also [12 for a bijection with unconstrained triangulations. This bijection has, truth to tell, brought about our discovery of transversal edge-partitions. Indeed, it turns out to "transport" a so-called transversal edge-bicoloration of a ternary tree into the minimal transversal edge-partition of its associated triangulation, in the same way that bijection of [12 transports the structure of Schnyder woods. In addition, the bijection gives a combinatorial way to enumerate rooted 4-connected triangulations, which were already counted by Tutte in [17] using algebraic methods.

\section{Definition of Transversal Structures}

\subsection{Transversal Edge-Partition}

Let $T$ be an irreducible triangulation of the 4-gon. Edges and vertices of $T$ are said inner or outer whether they belong to the outer face or not. A transversal edge-partition of $T$ is a partition of the inner edges of $T$ into two sets, say in blue and in red edge, such that the following conditions are satisfied.

- C1 (Inner vertices): In clockwise order around each inner vertex, its incident edges form: a non empty interval of red edges, a non empty interval of blue edges, a non empty interval of red edges, and a non empty interval of blue edges, see Figure 1].

- C2 (Border vertices): Writing $a_{1}, a_{2}, a_{3}, a_{4}$ for the border vertices of $T$ in clockwise order, all inner edges incident to $a_{1}$ and to $a_{3}$ are of one color and all inner edges incident to $a_{2}$ and to $a_{4}$ are of the other color.

Figure 1 gives an example of transversal edge-partition, where we use DARK RED for red edges and LIGHT BLUE for blue edges (the same convention will be used for all figures).

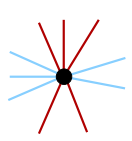

a)

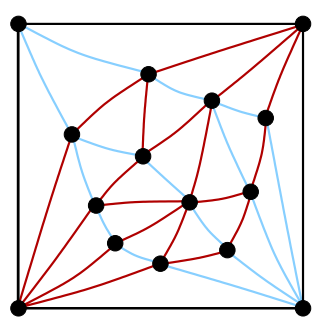

b)

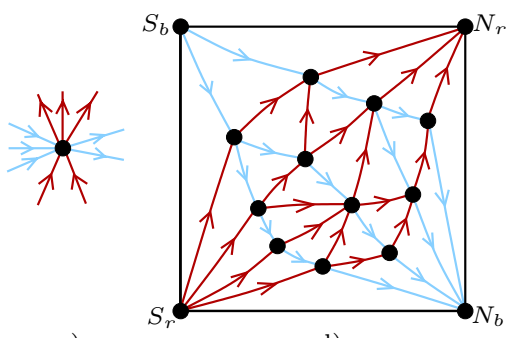

d)

Fig. 1. The structure of transversal edge-partition: local condition (a) and a complete example (b). In parallel, the structure of transversal couple of bipolar orientations: local condition (c) and a complete example (d). 


\section{$2.2 \quad$ Lattice Structure}

As it is the case with Schnyder Woods and bipolar orientations, the set of transversal edge-partitions of a fixed irreducible triangulation of the 4-gon is a distributive lattice. In addition, the "flip" operation has a nice geometric interpretation. To describe it, we have to introduce some terminology. Given $T$ an irreducible triangulation of the 4-gon endowed with a transversal edge-partition $X$, we define an alternating 4 -cycle as a 4 -cycle $\mathcal{C}$ of inner edges $\left(e_{1}, e_{2}, e_{3}, e_{4}\right)$ of $T$ which are color-alternating (i.e. two adjacent edges of $\mathcal{C}$ have different colors). The cycle $\mathcal{C}$ is called essential if its interior does not properly contain the interior of another alternating 4-cycle. Given a vertex $v$ of $\mathcal{C}$, we call left-edge (resp. right-edge) of $v$ the edge of $\mathcal{C}$ starting from $v$ and having the exterior of $\mathcal{C}$ on its left (resp. on its right). It can easily be proven that two cases can occur for $\mathcal{C}$ : either all edges interior to $\mathcal{C}$ and incident to a border vertex $v$ of $\mathcal{C}$ have the color of the left-edge of $v$, then $\mathcal{C}$ is called a left alternating 4-cycle; or all edges interior to $\mathcal{C}$ and incident to a border vertex $v$ of $\mathcal{C}$ have the color of the right-edge of $v$, then $\mathcal{C}$ is called a right alternating 4-cycle.

Theorem 1. Let $T$ be an irreducible triangulation of the 4-gon. Then the set $\mathcal{E}$ of transversal edge-partitions of $T$ is a non-empty distributive lattice. Given $X \in \mathcal{E}$, the flip operation consists in finding a right alternating 4 -cycle $\mathcal{C}$ of $X$ and then switching the colors of all edges interior to $\mathcal{C}$, making $\mathcal{C}$ a left alternating 4-cycle. The (unique) transversal edge-partition of $T$ without right alternating 4-cycle is said minimal.

Proof. The non emptiness of $\mathcal{E}$ will be proven constructively in Section 2.4 by providing an algorithm computing the minimal transversal edge-partition of $T$. The lattice structure follows from the fact that $\mathcal{E}$ is in bijection with the set of orientations of an associated graph (called the angular graph) where each vertex has a fixed outdegree. The set of such orientations with fixed outdegree is well-known to be a distributive lattice, see 6, 7.

\subsection{Transversal Couple of Bipolar Orientations}

Given a plane graph $G$ and two vertices $S$ (like South) and $N$ (like North) of $G$ incident to the outer face of $G$, a bipolar orientation of $G$ with poles $S$ and $N$ is an acyclic orientation of the edges of $G$ such that, for each vertex $v$ different from $S$ and $N$, there exists an oriented path from $S$ to $N$ passing by $v$, see [5] for a detailed decription.

Let $T$ be an irreducible triangulation of the 4-gon. Call $N_{r}, N_{b}, S_{r}$ and $S_{b}$ the 4 border vertices of $T$ in clockwise order around the outer face of $T$. A transversal couple of bipolar orientations is an orientation and a partition of the inner edges of $T$ into red and blue edges such that the following two conditions are satisfied (see Figure 11d for an example):

- C1' (Inner vertices): In clockwise order around each inner vertex of $T$, its incident edges form: a non empty interval of outgoing red edges, a non empty 
interval of outgoing blue edges, a non empty interval of ingoing red edges, and a non empty interval of ingoing blue edges, see Figure 1t.

- C2' (Border vertices): All inner edges incident to $N_{b}, N_{r}, S_{b}$ and $S_{r}$ are respectively ingoing blue, ingoing red, outgoing blue, and outgoing red.

This structure is also defined in [10] under the name of regular edge labeling. The following proposition explains the name of transversal couple of bipolar orientations and is also stated in [10]:

Proposition 1. Let $T$ be an irreducible triangulation of the 4-gon. Given a transversal couple of bipolar orientations of $T$, the (oriented) red edges induce a bipolar orientation of the plane graph obtained from $T$ by removing $S_{b}, N_{b}$, and all non red edges. Similarly, the blue edges induce a bipolar orientation of $T$ deprived from $S_{r}, N_{r}$ and all non blue edges.

Proposition 2. To each transversal couple of bipolar orientations of $T$ corresponds a transversal edge-partition of $T$, obtained by removing the orientation of the edges (Compare Figure 1] and Figure 1b). This correspondence is a bijection.

Proposition 2 allows us to manipulate equivalently transversal edge-partitions or transversal couples of bipolar orientations. The first point of view is more convenient to describe the lattice structure, the second one will be more convenient to describe the drawing algorithm in Section 3 .

\subsection{Algorithm Computing the Minimal Transversal Edge-Partition}

Let us now describe a simple iterative algorithm to compute transversal edgepartitions. Two different algorithms computing such transversal structures were already presented in [10. However we need to compute the minimal transversal edge-partition, to be used later in the straight-line drawing algorithm. During the execution, we also orient the edges, so that we compute in fact the underlying transversal couple of bipolar orientations. The algorithm we introduce consists in maintaining and iteratively shrinking a cycle $\mathcal{C}$ of edges of $T$ such that, in particular (we do not detail all invariants here):

- The cycle $\mathcal{C}$ contains the two edges $\left(S_{r}, S_{b}\right)$ and $\left(S_{r}, N_{b}\right)$.

- No edge interior to $\mathcal{C}$ connects two vertices of $\mathcal{C} \backslash\left\{S_{r}\right\}$

- All inner edges of $T$ outside of $\mathcal{C}$ are colored and oriented such that Innervertex Condition C1' (see Section 2) is satisfied for each inner vertex of $T$ outside of $\mathcal{C}$.

We initialize the cycle $\mathcal{C}$ with vertices $S_{r}, S_{b}, N_{b}$ and all interior neighbours of $N_{r}$, color in red all inner edges incident to $N_{r}$ and orient them toward $N_{r}$, see Figure 2b. Observe also that vertices of $\mathcal{C}$ different from $S_{r}$ can be ordered from left to right with $S_{b}$ as leftmost and $N_{b}$ as rightmost vertex. For two vertices $v$ and $v^{\prime}$ of $\mathcal{C} \backslash\left\{S_{r}\right\}$ with $v$ on the left of $v^{\prime}$, we write $\left[v, v^{\prime}\right]$ for the unique path on $\mathcal{C}$ that goes from $v$ to $v^{\prime}$ without passing by $S_{r}$.

To explain how to update $($ shrink) $\mathcal{C}$ at each step, we need a few definitions. An internal path of $\mathcal{C}$ is a path $\mathcal{P}$ of edges interior to $\mathcal{C}$ and connecting two vertices 
$v$ and $v^{\prime}$ of $\mathcal{C}$. We write $\mathcal{C}_{\mathcal{P}}$ for the cycle constituted by the concatenation of $\mathcal{P}$ and $\left[v, v^{\prime}\right]$. The path $\mathcal{P}$ is said eligible if the following conditions are satisfied:

- The paths $\mathcal{P}$ and $\left[v, v^{\prime}\right]$ have both at least one vertex different from $v$ and $v^{\prime}$.

- Each edge interior to $\mathcal{C}_{\mathcal{P}}$ connects a vertex of $\mathcal{P} \backslash\left\{v, v^{\prime}\right\}$ to a vertex of $\left[v, v^{\prime}\right] \backslash\left\{v, v^{\prime}\right\}$. In particular, the interior of $\mathcal{C}_{\mathcal{P}}$ contains no vertex.

- The cycle $\mathcal{C}^{\prime}$ obtained from $\mathcal{C}$ by replacing $\left[v, v^{\prime}\right]$ by $\mathcal{P}$ is such that no interior edge of $\mathcal{C}^{\prime}$ connects two vertices of $\mathcal{C}^{\prime} \backslash\left\{S_{r}\right\}$.

The update operation is the following: find an eligible internal path $\mathcal{P}$ of $\mathcal{C}$ and write $v$ and $v^{\prime}$ for its extremities with $v$ on the left of $v^{\prime}$ (so that $v$ and $v^{\prime}$ are called respectively left and right extremity of $\mathcal{P}$ ); then, color each internal edge of $\mathcal{C}_{\mathcal{P}}$ in red and orient it toward $\left[v, v^{\prime}\right] \backslash\left\{v, v^{\prime}\right\}$. Color all edges of $\left[v, v^{\prime}\right]$ in blue and orient them from $v$ to $v^{\prime}$; finally update $\mathcal{C}$ by replacing in $\mathcal{C}$ the path $\left[v, v^{\prime}\right]$ by the path $\mathcal{P}$.

It can easily be shown that the absence of non empty triangle on $T$ ensures that the algorithm terminates, i.e. that at each step the cycle $\mathcal{C}$ has an eligible internal path and can be updated (shrinked). After the last update operation, $\mathcal{C}$ is empty. Using all invariants of colors and orientations of edges satisfied by $\mathcal{C}$, it can be shown that the obtained orientation and coloration of inner edges of $T$ is a transversal couple of bipolar orientations. Figure 2 illustrates the complete execution of the algorithm on an example.

This algorithm can easily be adapted to give an algorithm, called ComPUTEMinimal(T), which computes the transversal couple of bipolar orientations associated (by removing orientation of edges) to the minimal transversal edge-partition of $T$, as defined in Theorem 1. Observe that, at each step of the algorithm, eligible paths of $\mathcal{C}$ can be ordered from left to right, by saying that $\mathcal{P}_{1} \geq \mathcal{P}_{2}$ if the left extremity and the right extremity of $\mathcal{P}_{1}$ are (weakly) on the
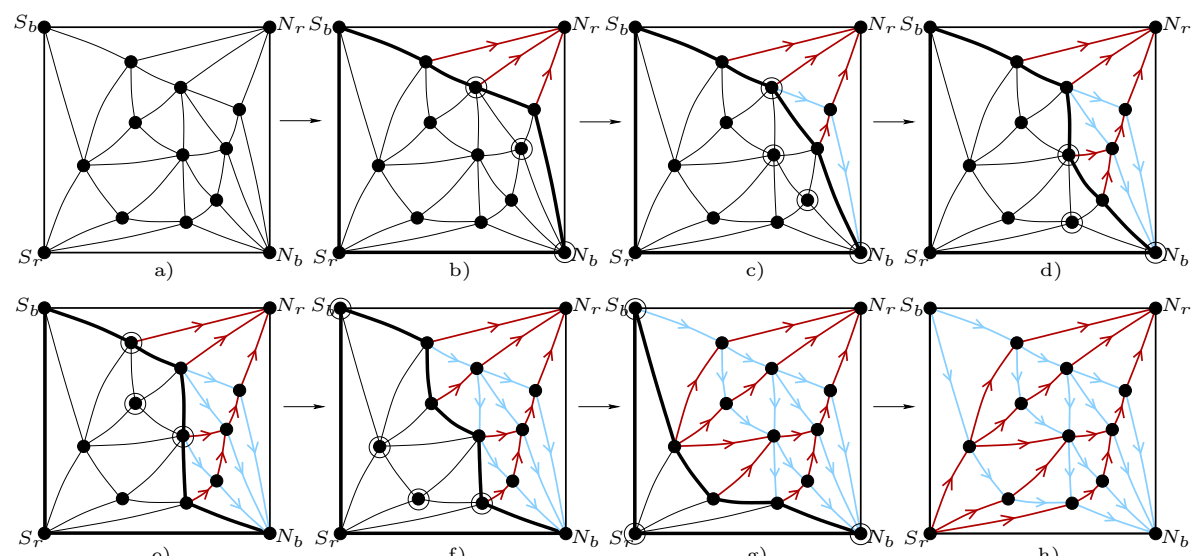

Fig. 2. An example of execution of the algorithm computing the minimal transversal couple of bipolar orientations. Vertices of the rightmost eligible path are surrounded. 
left respectively of the left extremity and of the right extremity of $\mathcal{P}_{2}$. Although this order is only partial, it can easily be shown to admit a unique minimum, called rightmost eligible path of $\mathcal{C}$. Algorithm Computeminimal(T) consists in choosing the rightmost eligible path at each step of the iterative algorithm described above, see also Figure 2, where the execution respects this choice.

Proposition 3. Given an irreducible triangulation $T$ of the 4-gon, Algorithm ComputeMinimal (T) outputs the transversal couple of bipolar orientations associated to the minimal transversal edge-partition of $T$ (by removing edge orientations). In addition, ComputeMinimal(T) can be implemented to run in linear time.

\section{Application to Straight-Line Drawing}

We recall that a straight line drawing of a plane graph $G$ consists in placing all points of $G$ on a regular grid of size $[0, W] \times[0, H]$ and then linking each pair of adjacent vertices of $G$ by a segment, with the condition that two different segments can only meet at their endpoints. The integers $W$ and $H$ are called the width and the height of the grid.

The structure of transversal edge-partition can be used to derive a simple algorithm, called TrAnsversalDrAw, to perform straight line drawing of an irreducible triangulation $T$ of the 4 -gon. First we have to give a few definitions. The plane graph obtained from $T$ by removing all blue (resp. red) edges is called the red-map (resp. blue-map) of $T$ and is denoted by $T_{r}$ (resp. $T_{b}$ ). We write $f_{r}$ and $f_{b}$ for the number of inner faces of $T_{r}$ and $T_{b}$. Given an inner vertex $v$ of $T$, we define the leftmost outgoing red path of $v$ as the oriented path starting from $v$ and such that each edge of the path is the leftmost outgoing red edge at its origin. As the orientation of red edges is bipolar, this path has no cycle and ends at $N_{r}$. We also define the rightmost ingoing red path of $v$ as the path starting from $v$ and such that each edge of the path is the rightmost ingoing red edge at its extremity. This path is also acyclic and ends at $S_{r}$. We call separating red path of $v$ the concatenation of these two paths and denote it by $\mathcal{P}_{r}(v)$. The path $\mathcal{P}_{r}(v)$ goes from $S_{r}$ to $N_{r}$ passing by $v$, and separates inner faces of $T_{r}$ into two sets: those on the left of $\mathcal{P}_{r}(v)$ and those on the right of $\mathcal{P}_{r}(v)$. Similarly, we define the leftmost outgoing blue path, the rightmost ingoing blue path, and write $\mathcal{P}_{b}(v)$ for their concatenation, called separating blue path of $v$.

Algorithm TransversalDraw consists of the following steps, see Figure 3 for a complete execution:

- Perform Computeminimal(T) to endow $T$ with its minimal transversal couple of bipolar orientations.

- Take a regular grid of width $f_{r}$ and height $f_{b}$.

- Place the border vertices $S_{r}, S_{b}, N_{b}, N_{r}$ respectively at coordinates $(0,0)$, $\left(0, f_{b}\right),\left(f_{r}, 0\right)$ and $\left(f_{r}, f_{b}\right)$.

- For each inner vertex $v$ of $T$, place $v$ on the grid in the following way:

- The abscissa of $v$ is the number of inner faces of $T_{r}$ on the left of $\mathcal{P}_{r}(v)$.

- The ordinate of $v$ is the number of inner faces of $T_{b}$ on the right of $\mathcal{P}_{b}(v)$. 


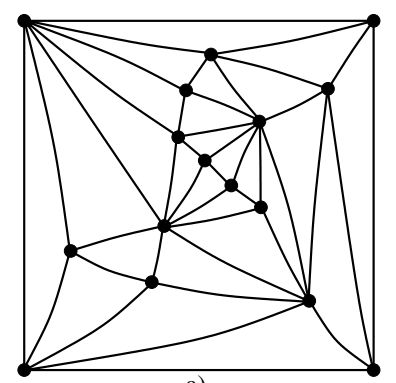

a)

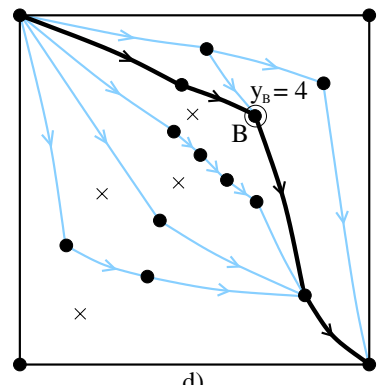

d)

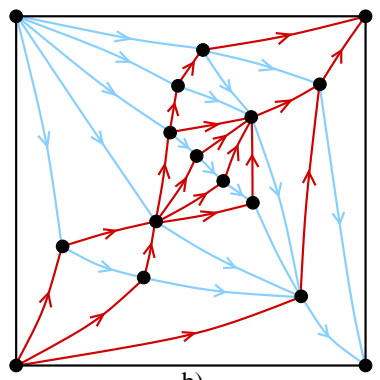

b)

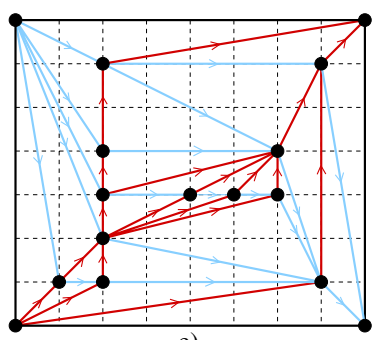

e)

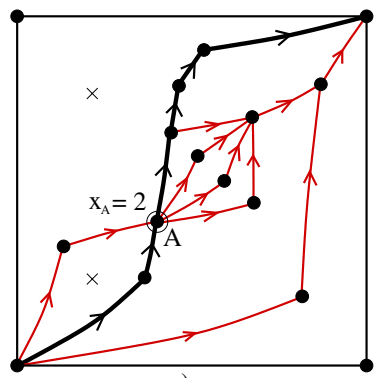

c)

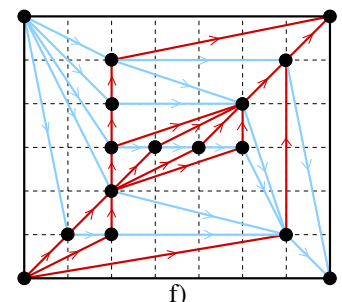

Fig. 3. The execution of Algorithm TransversalDraw (a)-(e), and of Algorithm CompactTransversalDraw (a)-(f) on an example

Algorithm TransversalDraW can be enhanced into an algorithm, called COMPACTTRANSVERsalDraw, giving a more compact drawing. The further step consists in deleting the unused abscissas and ordinates of the drawing computed by TransversalDraw. An example is given on Figure 3 d, obtained from Figure 3 c after having deleted the unused abscissa 3 and the unused ordinate 5 .

Theorem 2. Algorithm TRAnsversalDraw and Algorithm CompaCtTRANS VERSALDRAW can be implemented to run in linear time and compute a straight line drawing of an irreducible triangulation $T$ of the 4-gon such that:

- All red edges are oriented from bottom to top and weakly oriented from left to right.

- All blue edges are oriented from left to right and weakly oriented from top to bottom.

- If $T$ has $n$ vertices, then the width $W$ and height $H$ of the grid of the drawing given by TransversalDraW $(T)$ verify $W+H=n-1$.

- Let $T$ be taken uniformly at random among irreducible triangulations of the 4-gon with $n$ vertices. The width $W_{c}$ and the height $H_{c}$ of the grid of the drawing output by COMPACTTRANSVERSALDRAW(T) are asymptotically almost surely equal to $\frac{11}{27} n$, up to fluctuations $\epsilon_{W_{c}}$ and $\epsilon_{H_{c}}$ of order $\sqrt{n}$.

In fact the transversal structure used to give coordinates to vertices need not to be the minimal one. Using any other transversal couple of bipolar orientations, 
the three first points of Theorem 2 remain true. However the analysis of the reduction-factor $\frac{5}{27}$ with COMPACTTRANSVERSALDRAW $(\mathrm{T})$ crucially requires that the transversal structure is the minimal one, see Section 4.2 .

Corollary 1. Each 4-connected plane graph $G$ with $n$ vertices and at least 4 vertices on the outer face can be embedded with a straight-line drawing on a regular grid $W \times H$ with $W+H \leq n-1$.

\section{Bijection with Ternary Trees and Applications}

\subsection{Description of the Bijection}

A ternary tree $A$ is a tree embedded in the plane with nodes of degree 4 , called inner nodes and nodes of degree 1, called leaves. Edges of $A$ connecting two inner nodes are called inner edges and edges incident to a leaf are called stems (these are "pending" edges). A ternary tree can be rooted by marking one of its leaves, and such rooted ternary trees correspond to the classical definition of ternary trees (i.e. all nodes have either 0 or 3 children).

We describe briefly the bijection (see [8, 12, for detailed descriptions of similar bijections), consisting of three main steps: local closure, partial closure and complete closure. Perform a counterclockwise traversal of $A$ (imagine an ant walking around $A$ with the infinite face on its right). If a stem $s$ and then two inner edges $e_{1}$ and $e_{2}$ are successively encountered during the traversal, merge the extremity of $s$ with the extremity of $e_{2}$, so as to close a triangular face. This operation is called local closure, see Figure $4 \mathrm{~b}$. Now we can restart a counterclockwise traversal around the new Figure $F$, which is identical to $A$, except that it contains a triangular face and, more important, the stem $s$ has become an inner edge. Each time we find a succession (stem, edge, edge), we perform a local closure, update the figure, and restart, until no local closure is possible. This greedy execution of local closures is called the partial closure of $A$, see Figure 4t. It can easily be shown that the figure $F$ obtained by partial closure of $A$ does not depend of the order of execution of the local closures. Finally, the last step, called complete closure (see Figure 4d), consists in drawing a 4-gon, and then merging the extremity of each unmatched stem with a border vertex, so as to create only

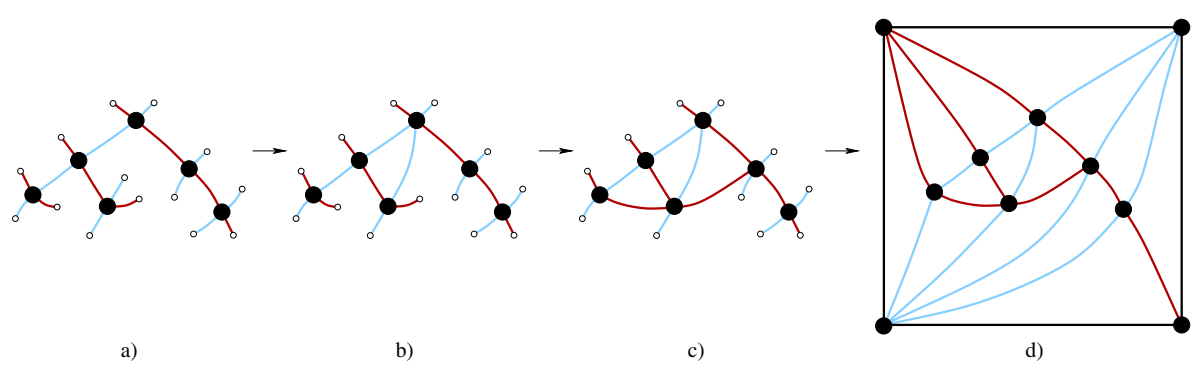

Fig. 4. The execution of the closure on an example 
triangular inner faces. It can be shown that the choice of an outer 4-gon is the good one so that this last operation works without conflict.

Observe that the edges of a ternary tree $A$ can be bicolored in blue and red edges so that two successive edges incident to an inner node of $A$ have always different color, see Figure 4 a. This bicoloration, unique up to the choice of the colors, is called the transversal edge-bicoloration of $A$. Observe that Inner Vertex Condition $\mathrm{C} 1$ is satisfied on $A$ and remains satisfied throughout the closure.

Theorem 3. The closure is a bijection between ternary trees with $n$ inner nodes and irreducible triangulations of the 4-gon with $n$ inner vertices.

The closure transports the transversal edge-bicoloration of a ternary tree into the minimal transversal edge-partition of its image.

Proof. Injectivity can easily be proven by uniqueness of the transversal edgepartition without right alternating 4-cycle. The inverse of the closure consists in computing the minimal transversal edge-partition of $T$ and using the colors to remove some half-edges, so as to leave a ternary tree.

An irreducible triangulation of the 4 -gon is rooted by choosing one of its 4 border edges and orienting this edge with the infinite face on its right. This well-known operation eliminates symmetries of the triangulation.

Corollary 2. The closure induces a 4-to- $(2 n+2)$ correspondence between the set $\mathcal{A}_{n}$ of rooted ternary trees with $n$ inner nodes and the set $\mathcal{T}_{n}$ of rooted irreducible triangulations of the 4-gon with $n$ inner vertices.

As an enumerative consequence, $\left|\mathcal{T}_{n}\right|=\frac{4}{2 n+2}\left|\mathcal{A}_{n}\right|=\frac{4(3 n) !}{(2 n+2) ! n !}$.

Proof. The proof follows easily from the bijection stated in Theorem 3 and from the fact that a ternary tree with $n$ inner nodes has $2 n+2$ leaves and an object of $\mathcal{T}_{n}$ has 4 edges (the 4 border edges) to carry the root.

\subsection{Applications}

The closure-bijection has several applications. A first one is a linear-time algorithm to perform uniform random sampling of objects of $\mathcal{T}_{n}$, using the fact that rooted ternary trees with $n$ inner nodes can readily be uniformly sampled using parenthesis words. A thorough study of such sampling algorithms is given in [14. In addition, sampled objects of $\mathcal{T}_{n}$ are naturally endowed, through the closure, with their minimal transversal edge-partition. Hence, we can easily run face-counting algorithms TransversalDraw and COMPACTTRAnsverSALDRAW on the sampled objects. Performing simulations on objects of large size $(n \approx 50000)$, it was observed that the size of the grid is always approximately $\frac{n}{2} \times \frac{n}{2}$ with TRANSVERSALDRAW and $\frac{n}{2}(1-\alpha) \times \frac{n}{2}(1-\alpha)$ with COMPACTTRANSVERSALDRAW, where $\alpha \approx 0.18$. It turns out that the size of the grid can be readily analyzed thanks to our closure-bijection, in the same way that bijection of [12 allowed to analyze parameters of Schnyder woods in 3]. Indeed, 

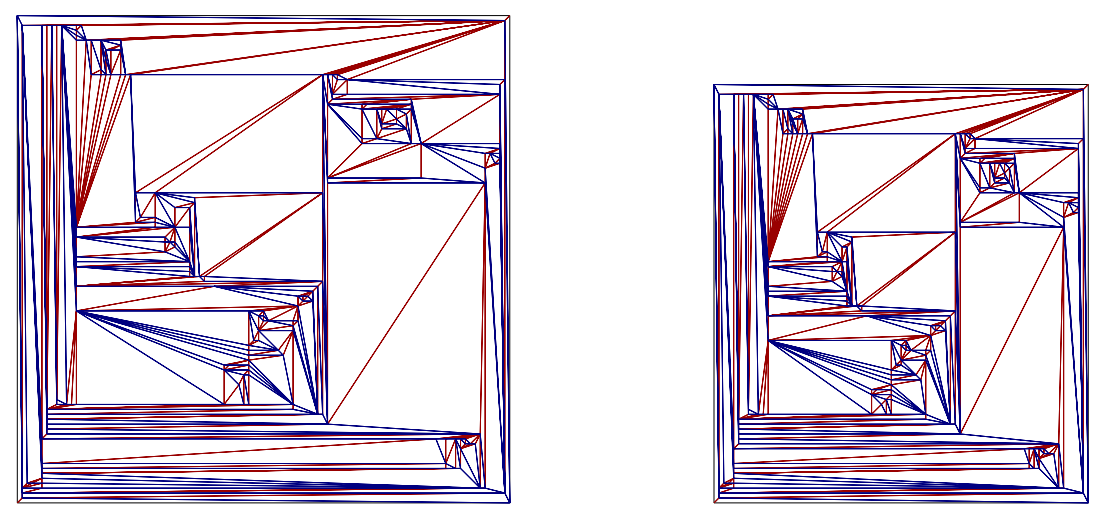

Fig. 5. A random triangulation with 200 vertices embedded with Algorithms TranSVERSALDraW and COMPACTTransversalDraW

unused abscissas and ordinates of TRANSVERSALDRAW correspond to certain inner edges of the ternary tree, whose number can be proven to be asymptotically almost surely $\frac{5 n}{27}$ up to fluctuations of order $\sqrt{n}$.

A second application is counting rooted 4-connected triangulations with $n$ vertices, whose set is denoted by $\mathcal{C}_{n}$. It is well known that a 4 -connected triangulation is a triangulation where each 3-cycle delimits a face. Hence, the operation of removing the root edge of an object of $\mathcal{C}_{n}$ and carrying the root on the counterclockwise-consecutive edge is an (injective) mapping from $\mathcal{C}_{n}$ to $\mathcal{T}_{n-4}$. However, given $T \in \mathcal{T}_{n-4}$, the inverse edge-adding-operation can create a separating 3 -cycle if there exists an internal path of length 2 connecting the origin of the root of $T$ to the vertex diametrically opposed in the outer face of $T$. Objects of $\mathcal{T}_{n-4}$ having no such internal path are said undecomposable and their set is denoted by $\mathcal{U}_{n-4}$. The above discussion ensures that they are in bijection with $\mathcal{C}_{n}$. A maximal decomposition of an object $T$ of $\mathcal{T}$ along the above mentioned interior paths of length 2 ensures that $T$ is a sequence of objects of $\mathcal{U}$. After a few simple manipulations and using Corollary 2, we get:

Proposition 4. The series $C(z)$ counting rooted 4-connected triangulations by their number of inner vertices has the following expression:

$$
C(z)=\frac{z\left(A(z)-A(z)^{2}+1\right)}{1+z\left(A(z)-A(z)^{2}+1\right)}
$$

where $A(z)=z(1+A(z))^{3}$ is the series counting rooted ternary trees by their number of inner nodes.

Acknowledgments. I would like to thank my advisor Gilles Schaeffer. He has greatly helped me to produce this work through numerous discussions, steady encouragment and useful suggestions. I also thank Nicolas Bonichon for fruitful discussions and Thomas Pillot for very efficient implementations of all algorithms presented in this extended abstract. 


\section{References}

1. Therese C. Biedl, Goos Kant, and Michael Kaufmann. On triangulating planar graphs under the four-connectivity constraint. Algorithmica, 19(4):427-446, 1997.

2. N. Bonichon, S. Felsner, and M. Mosbah. Convex drawings of 3-connected plane graphs. In GD '04: Proceedings of the Symposium on Graph Drawing, pages 287299. Springer-Verlag, 2004.

3. N. Bonichon, C. Gavoille, N. Hanusse, D. Poulalhon, and G. Schaeffer. Planar graphs, via well-orderly maps and trees. In $30^{\text {th }}$ International Workshop, Graph Theoretic Concepts in Computer Science (WG), volume 3353 of Lecture Notes in Computer Science, pages 270-284. Springer-Verlag, 2004.

4. H. de Fraysseix, P. Ossona de Mendez, and J. Pach. Representation of planar graphs by segments. Intuitive Geometry, 63:109-117, 1991.

5. H. de Fraysseix, P. Ossona de Mendez, and P. Rosenstiehl. Bipolar orientations revisited. Discrete Appl. Math., 56(2-3):157-179, 1995.

6. P. O. de Mendez. Orientations bipolaires. PhD thesis, Paris, 1994.

7. Stefan Felsner. Lattice structures from planar graphs. Electronic Journal of Combinatorics, (R15):24p., 2004.

8. É. Fusy, D. Poulalhon, and G. Schaeffer. Dissections and trees, with applications to optimal mesh encoding and to random sampling. In 16th Annual ACM-SIAM Symposium on Discrete Algorithms, January 2005.

9. X. He. Grid embedding of 4-connected plane graphs. In GD '95: Proceedings of the Symposium on Graph Drawing, pages 287-299. Springer-Verlag, 1996.

10. G. Kant and Xin He. Regular edge labeling of 4-connected plane graphs and its applications in graph drawing problems. Theoretical Computer Science, 172(12):175-193, 1997.

11. K. Miura, S. Nakano, and T. Nishizeki. Grid drawings of four-connected plane graphs. Disc. Comput. Geometry, 26(2):73-87, 2001.

12. D. Poulalhon and G. Schaeffer. Optimal coding and sampling of triangulations. In Automata, Languages and Programming. 30th International Colloquium, ICALP 2003, Eindhoven, The Netherlands, June 30 - July 4, 2003. Proceedings, volume 2719 of Lecture Notes in Computer Science, pages 1080-1094. Springer-Verlag, 2003.

13. G. Schaeffer. Conjugaison d'arbres et cartes combinatoires aléatoires. PhD thesis, Université Bordeaux I, 1998.

14. G. Schaeffer. Random sampling of large planar maps and convex polyhedra. In Annual ACM Symposium on Theory of Computing (Atlanta, GA, 1999), pages 760-769 (electronic). ACM, New York, 1999.

15. W. Schnyder. Planar graphs and poset dimension. Order, 5:323-343, 1989.

16. W. Schnyder. Embedding planar graphs on the grid. In SODA '90: Proceedings of the first annual ACM-SIAM symposium on Discrete algorithms, pages 138-148, 1990.

17. W. T. Tutte. A census of planar triangulation. Canad. J. Math., 14:21-38, 1962. 